\title{
金属苯炔的合成与化学性质
}

\author{
陈江溪何国梅贾国成* \\ (香港科技大学化学系 香港)
}

\begin{abstract}
摘要 金属苯炔是一类新颖的杂环芳香化合物. 它们可看成是苯炔分子中的一个碳原子被等瓣的过渡金属基团取代而 衍生出来的六元杂环化合物. 近年来, 金属苯炔的化学引起了人们的兴趣和关注. 一系列含锇和铼的金属苯炔已被成 功地合成和鉴定. 这些金属苯炔不仅具有有机化合物的芳香性，还具有金属有机化合物的属性. 它们既可以发生芳香 体系的经典反应(如亲电取代反应), 也可以发生金属有机化合物的反应(如卡宾化合物的形成).

关键词 芳香性; 金属有机化合物; 金属苯炔; 金属苯
\end{abstract}

\section{Synthesis and Chemical Properties of Metallabenzynes}

\author{
Chen, Jiangxi He, Guomei Jia, Guochen* \\ (Department of Chemistry, Hong Kong University of Science and Technology, Hong Kong)
}

\begin{abstract}
Metallabenzynes are an interesting class of metallaraomatics. They can be regarded as transition-metal analogs of benzyne in which a $\mathrm{C}$ atom is replaced by an isolobal transition metal fragment. The chemistry of this class of compounds has received much recent attention. A series of metallabenzynes containing osmium and rhenium have been isolated. It has also been demonstrated both experimentally and theoretically that metallabenzynes can have properties of both aromatic organic compounds and organometallic compounds. For example, metallabenzynes, like organic aromatic compounds, could undergo electrophilic substitution reactions to give new metallabenzynes; react with nucleophiles to generate metallabenzene species through addition of nucleophiles at the carbyne carbons of metallabenzynes and undergo reductive elimination to give carbene complexes.
\end{abstract}

Keywords aromaticity; organometallic compounds; metallabenzynes; metallabenzenes

一个多世纪以来, 芳香性一直是有机化学的研究热 点之一, 它至今仍吸引着无数全球化学家们的热情并为 之努力. 在众多的芳香化合物中, 最具典型和传奇的化 合物就是苯. 当苯分子中的一个 $\mathrm{CH}$ 基团被等瓣的杂原 子或基团取代时，所得到的化合物被称为杂环芳香化合 物. 例如, $\mathrm{N}$ 原子和 $\mathrm{CH}$ 基团具有相似的前线轨道和相同 的价电子数, 它们是等瓣相似的. 把苯分子中的一个 $\mathrm{CH}$ 基团用等瓣的 $\mathrm{N}$ 原子取代后可得到吡啶，吡啶是一 个典型的含主族元素的杂环芳香化合物. 百多年来, 已 有非常多含主族元素或基团的杂环芳香化合物被合成 出来, 尤其是六元杂环芳香化合物 ${ }^{[1]}$. 这些已知的含主 族元素或基因的六元杂环芳香化合物主要为由 $\mathrm{O}^{+}, \mathrm{S}^{+}$, $\mathrm{Se}^{+}, \mathrm{Te}^{+}, \mathrm{N}, \mathrm{P}^{[2]}, \mathrm{As}^{[3]}, \mathrm{Sb}^{[3]}, \mathrm{Bi}^{[3]}, \mathrm{SiR}^{[4,5]}, \mathrm{SnR}^{[6]}, \mathrm{BR}^{-[7]}$ 和

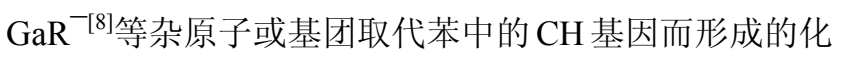
合物.
与此类似，金属苯是把苯分子中 $\mathrm{CH}$ 基团用等瓣的 金属基团取代而衍生出来的六元杂环芳香化合物. 近几 十年来, 含过渡金属的金属苯化学引起了化学家们的极 大兴趣和关注. 人们之所以对含过渡金属的金属苯感兴 趣是因为它们不仅具有有机化合物的芳香性, 还可能具 有金属有机化合物的属性. 早在 1979 年 Hoffman 等 ${ }^{[9]}$ 通过理论计算预测含过渡金属的金属苯具有一定的稳 定性, 因此它们有可能被合成和分离出来. 1982 年, Roper 等 ${ }^{[10]}$ 报道了首例稳定的含过渡金属锇的金属苯合 成及鉴定. 从那之后, 含过渡金属的金属苯化学研究, 不管在理论方面, 还是实验方面, 都得到了蓬勃的发展. 有关含过渡金属的金属苯化学的进展, 在一些综述内已 有详细的论述, 其中包括 2001 年 Bleeke ${ }^{[1]}$ 的综述, 2004 年夏海平等 ${ }^{[12]}$ 的综述, 2006 年 $\mathrm{Wright}^{\left[{ }^{[1]}\right]}$ 的综述, 以及 2006 年 Haley 等 ${ }^{[14]}$ 的综述. 此外, 还有两篇关于铱苯化

* E-mail: chjiag@ust.hk

Received March 29, 2013; revised April 12, 2013; published online April 15, 2013.

Project supported by the Hong Kong Research Grant Council (Nos. HKUST 602611, 601812).

香港研究资助局基金(Nos. HKUST 602611, 601812)资助项目. 
学的短评, 一篇发表于 1991 年 ${ }^{[15]}$, 另一篇发表于 2007 年 ${ }^{[16]}$.

金属苯炔是同金属苯紧密相关的一类化合物. 它们 可看成是苯炔分子中的一个碳原子被等瓣相似的过渡 金属基团取代而衍生出来的六元杂环芳香化合物 ${ }^{[17]}$. 2001 年我们 ${ }^{[18]}$ 报道了首例稳定的含过渡金属的金属苯 炔的合成及表征. 近年来, 我们及夏海平的研究组在金 属苯炔化学方面继续开展了工作, 并成功地合成和鉴定 了一系列金属苯炔, 也同时探索了金属苯炔的相关化学 性质 ${ }^{[19]}$. 关于金属苯炔化学的早期进展已总结在 2004 年的一篇短评, 2006 年一篇关于金属苯化学的综述 ${ }^{[14]}$ 和 2007 年一篇关于锇卡拜配合物化学的综述 ${ }^{[20]}$ 中. 最近 几年来, 金属苯炔的化学又有了更进一步的发展. 在本 文中, 我们将主要总结我们在金属苯炔化学方面的研究 进展, 特别是有关金属苯炔的合成和化学性质方面的进 展.

\section{1 构筑金属苯炔环的方法}

目前已知的金属苯炔大部分是含锇的金属苯炔(即 锇苯炔). 锇苯炔化合物 2 是首例被分离出来的金属苯 炔, 它是由化合物 $\mathrm{OsCl}_{2}\left(\mathrm{PPh}_{3}\right)_{3}(\mathbf{1})$ 和 $\mathrm{HC} \equiv \mathrm{CSiMe}_{3}$ 在苯 中反应制得的(Scheme 1) ${ }^{[18]}$. 该反应还会生成包括三氯 锇卡拜化合物 $\mathbf{3}$ 及卡宾化合物 $\mathbf{4}$ 和 $\mathbf{5}$ 的其它产物. 但是, 将该方法拓展到用其它有机炔烃原料来制备相应的锇 苯炔化合物的尝试并未获得成功.

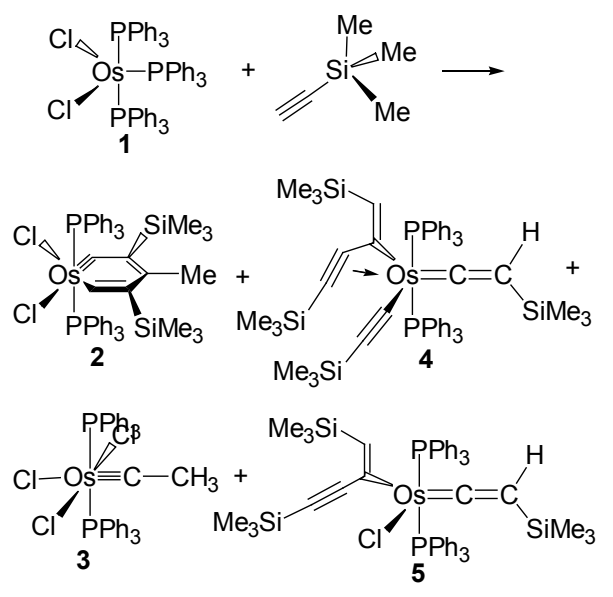

Scheme 1

2005 年, 我们 ${ }^{[21]}$ 报道了用卡宾化合物来合成锇苯 炔的方法. 卡宾化合物 $\mathbf{6}$ 与乙炔基金(I)的衍生物在盐酸 铵盐 $\left(\mathrm{NEt}_{3} \cdot \mathrm{HCl}\right)$ 的存在下反应可得到锇苯炔. 例如, 化 合物 6 和 $\left(\mathrm{PPh}_{3}\right) \mathrm{AuC} \equiv \mathrm{CPh}$ 反应，经中间体 11 及 15 得到 了锇苯炔 7 (Scheme 2). 在同样的反应条件下, 化合物 6 和 $\left(\mathrm{PPh}_{3}\right) \mathrm{AuC} \equiv \mathrm{CSiMe}_{3}$ 反应可得到含 $\mathrm{SiMe}_{3}$ 的锇苯炔 $\mathbf{9}$; 化合物 6 和 $\left(\mathrm{PPh}_{3}\right) \mathrm{AuC} \equiv \mathrm{C}$ - $p$-tolyl 反应可得到含 $p$-tolyl
的锇苯炔 8 , 而化合物 6 和 $\left(\mathrm{PPh}_{3}\right) \mathrm{AuC} \equiv \mathrm{C}-n-\mathrm{Bu}$ 反应可得 到含 $n-\mathrm{Bu}$ 的锇苯炔 $\mathbf{1 0}$.
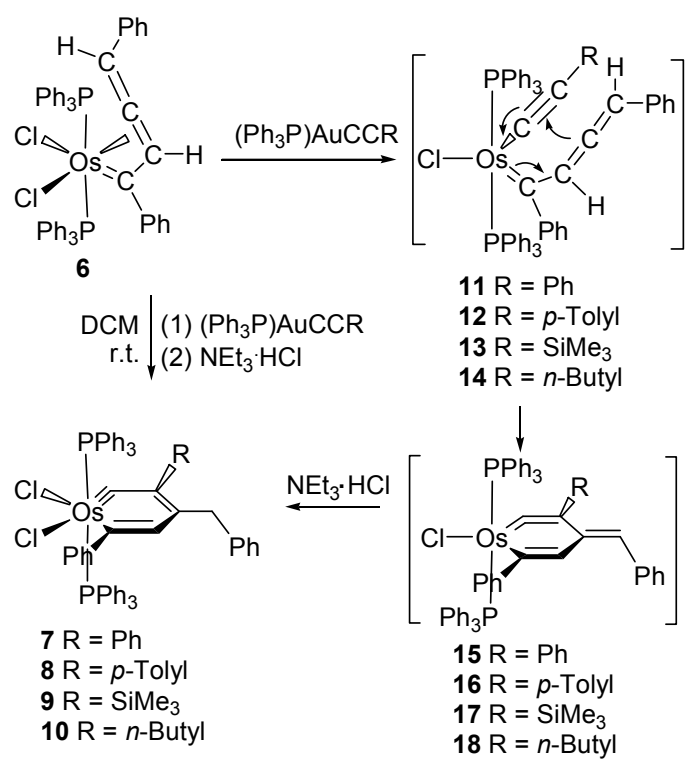

Scheme 2

最近，夏海平等 ${ }^{[22]}$ 发现也能从其它卡宾化合物来 合成锇苯炔. 例如, 卡宾化合物 19 与 $\mathrm{HC} \equiv \mathrm{CCH}(\mathrm{OEt})_{2}$ 反应得到了含 $\mathrm{PPh}_{3}$ 取代基的锇苯炔 20 . 锇苯炔 20 可能 是经过锇苯异构体 21 的异构化而生成的 (Scheme 3).

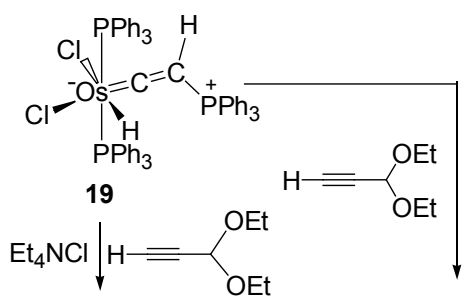

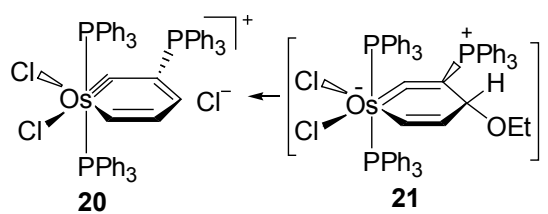

Scheme 3

到目前为止，最有效的合成锇苯炔的方法是通过锌 还原官能化的锇卡拜化合物的反应. 该方法最先被用于 锇菜炔化合物 24 的合成(Scheme 4) ${ }^{[23]}$. 卡拜化合物 22 和 23 的混合物与锌粉、三苯基膦在四氢呋喃中反应得 到了锇菜炔化合物 24 和帅基化合物 25 的混合物. 该反 应可能是经如下机理完成的: 混合物 22 和 23 先被锌还 原成 16 电子的四配位卡拜化合物 26, 化合物 26 发生 $\mathrm{C}-\mathrm{Cl}$ 键的氧化加成反应而生成含 $\mathrm{OsCl}_{2}$ 的锇荎炔化合 物 24; 化合物 26 也可发生 C-H 键的氧化加成反应而 得到含 $\mathrm{OsHCl}$ 的锇萗炔中间体 $\mathbf{2 7}$, 化合物 27 可发生还 


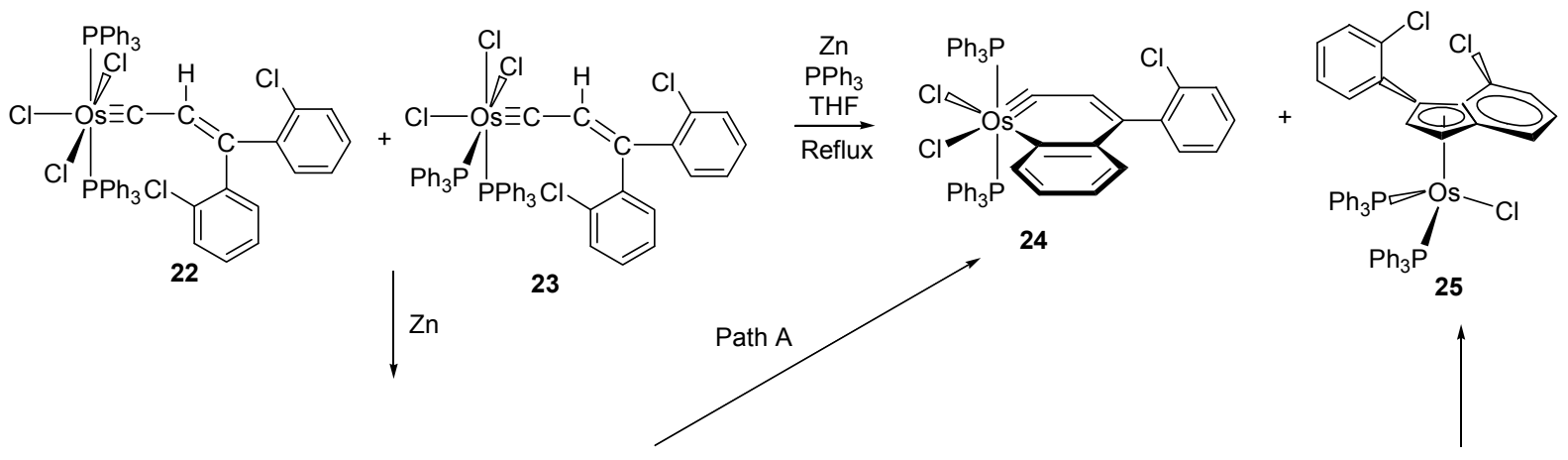

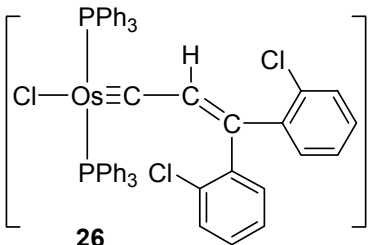

26

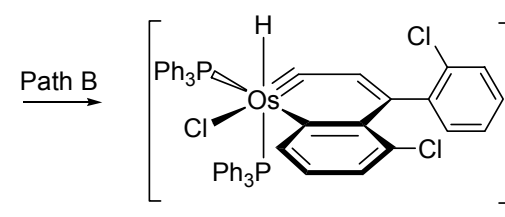

27

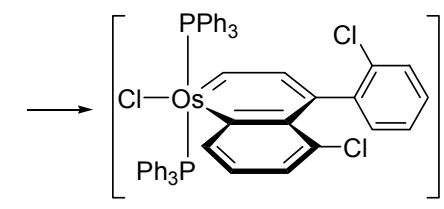

28

Scheme 4

原消去反应而得到锇萗苯化合物 $\mathbf{2 8}$, 化合物 $\mathbf{2 8}$ 再经过 还原消去反应而最终得到狮基化合物 25.

此方法可应用于制备其它的锇苯炔. 例如, 特丁基 取代的卡拜化合物 29 和 30 分别与锌在室温下反应生成 了相应的锇苯炔化合物 31 和 32. 在同样的反应条件下, 带金刚烷基的卡拜化合物 33 和 34 也会分别与锌粉反应 得到相应的锇苯炔化合物 35 和 36 (Scheme 5) ${ }^{[24]}$.

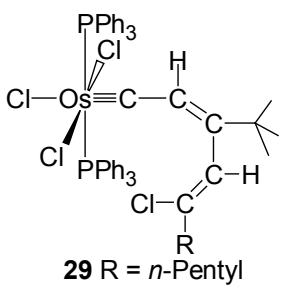
$30 \mathrm{R}=\mathrm{H}$

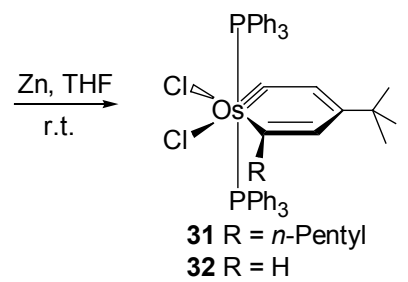

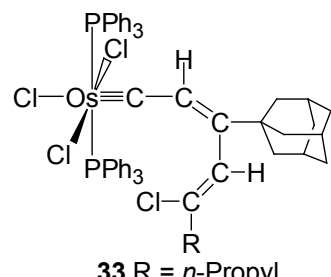

$33 \mathrm{R}=n-$ Propyl $34 \mathrm{R}=\mathrm{H}$

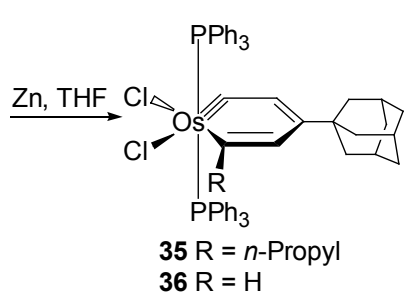

Scheme 5

最近，夏海平等 ${ }^{[25]}$ 报道了用含 $\mathrm{Os}-\mathrm{H}$ 的卡拜化合 物 37 来制备锇菜炔 38 的反应. 在 $\mathrm{O}_{2}$ 气氛下, 将化合物 37 在二氯乙烷中回流 $15 \mathrm{~h}$ 或者将化合物 37 的固体样品 在 $120{ }^{\circ} \mathrm{C}$ 加热 $5 \mathrm{~h}$ 均可得到锇荎炔化合物 38 (Scheme 6). 该反应可能是经过如下途径而完成的，化合物 37 可 把 $\mathrm{Os}-\mathrm{H}$ 上的 $\mathrm{H}$ 迁移到卡拜的碳原子上而形成一个锇 卡宾中间体, 再经 $\mathrm{C}-\mathrm{H}$ 键的氧化加成反应得到含 $\mathrm{Os}-$ $\mathrm{H}$ 的锇漛中间体 39. 中间体 39 再同 $\mathrm{O}_{2}$ 反应而得到锇荎
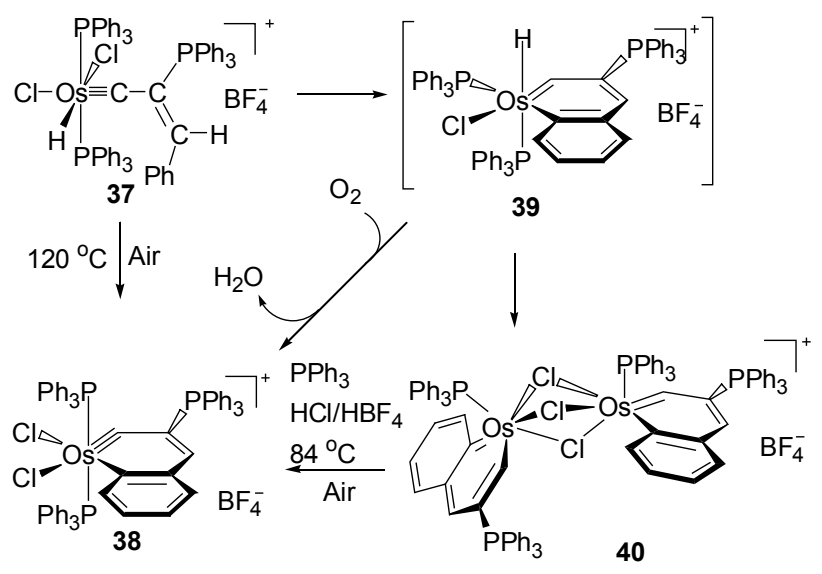

Scheme 6

炔 38. 有趣的是，在 $\mathrm{HCl}$ (1 equiv.), $\mathrm{HBF}_{4}$ (1 equiv.) 和过 量三苯基膦存在下, 在 $\mathrm{O}_{2}$ 气氛中把溶于二氯乙烷中的 锇萗二聚体 40 加热也可以得到锇萗炔化合物 38 .

目前只有 3 个锞苯炔化合物被成功合成出来 ${ }^{[26,27]}$, 它们是从化合物 $\mathrm{Re}\left\{\equiv \mathrm{CCH}=\mathrm{C}(\mathrm{R}) \mathrm{C} \equiv \mathrm{CSiMe}_{3}\right\} \mathrm{Cl}_{2}$ $\left(\mathrm{PMe}_{2} \mathrm{Ph}\right)_{3}(41, \mathrm{R}=t$-Butyl; 42, $\mathrm{R}=i$-Propyl; 43, $\mathrm{R}=1$ Adamantyl)制得的. 化合物 $\mathbf{4 1} \sim \mathbf{4 3}$ 可由 $\mathrm{ReH}_{5}\left(\mathrm{PMe}_{2}-\right.$ $\mathrm{Ph})_{3}$ 和炔醇 $\mathrm{HC} \equiv \mathrm{CC}(\mathrm{OH})(\mathrm{R}) \mathrm{C} \equiv \mathrm{CSiMe}_{3}\left(\mathrm{R}=\mathrm{CMe}_{3}\right.$, $\mathrm{CHMe}_{2}, 1$-Adamantyl) 在 $\mathrm{HCl}$ 存在下的反应制得(Scheme 7). 化合物 $\mathbf{4 1} \sim \mathbf{4 3}$ 与 $\mathrm{Me}_{3} \mathrm{CMgCl}$ 反应生成了含 $\mathrm{Re}-\mathrm{H}$ 的卡拜化合物 $\operatorname{Re}\left\{\equiv \mathrm{CCH}=\mathrm{C}(\mathrm{R}) \mathrm{C} \equiv \mathrm{CSiMe}_{3}\right\} \mathrm{HCl}-$ $\left(\mathrm{PMe}_{2} \mathrm{Ph}\right)_{3} \quad(47 \sim 49)$. 化合物 $47 \sim 49$ 应是由中间体 $\mathrm{Re}\left\{\equiv \mathrm{CCH}=\mathrm{C}(\mathrm{R}) \mathrm{C} \equiv \mathrm{CSiMe}_{3}\right\} \mathrm{Cl}\left(\mathrm{CMe}_{3}\right)\left(\mathrm{PMe}_{2} \mathrm{Ph}\right)_{3}(44 \sim$ 45)经过 $\beta-\mathrm{H}$ 消除反应而得到的. 化合物 $47 \sim 49$ 和 $n-\mathrm{Bu}_{4} \mathrm{NF}$ 反应形成了 $\mathrm{Re}\{\equiv \mathrm{CCH}=\mathrm{C}(\mathrm{R}) \mathrm{C} \equiv \mathrm{CH}\} \mathrm{HCl}-$ $\left(\mathrm{PMe}_{2} \mathrm{Ph}\right)_{3}(\mathbf{5 0} \sim \mathbf{5 2})$. 化合物 $\mathbf{5 0} \sim \mathbf{5 2}$ 在溶液中发生异构 
化反应先生成双环化合物 $\operatorname{Re}\{\mathrm{CH}=\mathrm{CH}-\mathrm{C}(\mathrm{R})=\mathrm{C}-$ $\mathrm{CH}=\} \mathrm{Cl}\left(\mathrm{PMe}{ }_{2} \mathrm{Ph}\right)_{3}(\mathbf{5 3} \sim \mathbf{5 5})$, 最终生成了铼苯炔化合物 $\mathrm{Re}\{\equiv \mathrm{CCH}=\mathrm{C}(\mathrm{R}) \mathrm{CH}=\mathrm{CH}\} \mathrm{Cl}\left(\mathrm{PMe}_{2} \mathrm{Ph}\right)_{3} \quad(\mathbf{5 6}, \mathrm{R}=t-\mathrm{Bu}-$ tyl; 57, $\mathrm{R}=i$-Propyl; 58, $\mathrm{R}=1$-Adamantyl).

\section{2 金属苯炔的反应性}

\section{1 亲电取代反应}

金属苯可以发生经典的芳香亲电取代反应. 例如, Os $\left\{\mathrm{C}_{5} \mathrm{H}_{4}(\mathrm{SMe})\right\} \mathrm{I}(\mathrm{CO})\left(\mathrm{PPh}_{3}\right)_{2}(\mathbf{5 9})^{[28]}$ 可以发生硝化反应生 成化合物 60, Ir $\left\{\mathrm{C}_{5} \mathrm{H}_{4}(\mathrm{SMe})\right\} \mathrm{Cl}_{2}\left(\mathrm{PPh}_{3}\right)_{2}(\mathbf{6 1})^{[29]}$ 可以发生 溴化反应生成化合物 62 (Scheme 8).

我们的实验证实金属苯炔和金属苯一样, 也能发生 芳香亲电取代反应. 金属苯炔能发生亲电取代反应的第 一个例子是金属锇苯炔与酸的反应 ${ }^{[30]}$. 在 $\mathrm{NaCl}$ 存在时, 金属锇苯炔化合物 $\mathbf{2}$ 和 $\mathrm{HBF}_{4} \cdot \mathrm{Et}_{2} \mathrm{O}$ 反应生成了中性的脱 三甲基硅基的锇苯炔化合物 63 (Scheme 9). 锇苯炔化合 物 63 也会和 $\mathrm{CF}_{3} \mathrm{SO}_{3} \mathrm{D}$ 发生 $\mathrm{H} / \mathrm{D}$ 交换反应生成在锇苯炔 环的间位碳上含氛的锇苯炔化合物 65. 如果没有 $\mathrm{NaCl}$ 的存在, 锇苯炔化合物 2 与 $\mathrm{HBF}_{4}$ 水溶液反应则得到离 子型的脱三甲基硅基的锇苯炔化合物 64 .
锇苯炔化合物 2 还能和其他的亲电试剂如 $\mathrm{Br}_{2}$, $\mathrm{NO}_{2} \mathrm{BF}_{4}, \mathrm{NOBF}_{4}, \mathrm{HCl} / \mathrm{H}_{2} \mathrm{O}_{2}$ 和 $\mathrm{AlCl}_{3} / \mathrm{H}_{2} \mathrm{O}_{2}$ 等在锇苯炔的 间位碳上发生亲电取代反应 ${ }^{[31]}$. 锇苯炔化合物 2 和过量 的 $\mathrm{Br}_{2}$ 反应的主产物是锇苯炔 $\mathrm{Os}\{\equiv \mathrm{CC}(\mathrm{Br})=$ $\left.\mathrm{C}\left(\mathrm{CH}_{3}\right) \mathrm{C}(\mathrm{Br})=\mathrm{CH}\right\} \mathrm{Br}_{2}\left(\mathrm{PPh}_{3}\right)_{2}$ (66, Scheme 10). 化合物 2 与 $\mathrm{NOBF}_{4}(1: 4), \mathrm{NaCl}$ 在 $-20 \sim-5{ }^{\circ} \mathrm{C}$ 间反应生成了一 个混合物, 我们可以从这个混合物中以 $72 \%$ 的产率分离 出化合物 67. 锇苯炔化合物 2 和过量的 $\mathrm{HCl} / \mathrm{H}_{2} \mathrm{O}_{2}$ 在 0 ${ }^{\circ} \mathrm{C}$ 反应可得到氯化产物 68 .

在 $\mathrm{NaCl}$ 存在下, 锇苯炔化合物 2 和 $\mathrm{NO}_{2} \mathrm{BF}_{4}(1: 4.5$ 物质的量的比), 在 $-16 \sim-5{ }^{\circ} \mathrm{C}$ 间反应生成了锇苯炔 化合物 69 和顺磁性卡宾化合物 70 (Scheme 10). 顺磁性 卡宾化合物 70 可能是通过如 Scheme 11 所示的机理而 形成的 ${ }^{[31]}$. $\mathrm{NO}_{2}^{+}$在化合物 2 的金属环的间位碳上发生 亲电取代反应得到锇苯炔化合物 71; 化合物 71 可发生 还原消除反应得到中间体 72; 其后转化为中间体 73; 中 间体 73 进一步转化成双自由基化合物 74; 最终, 双自 由基化合物 74 与溶剂发生自由基反应得到顺磁的化合 物 70. 该反应机理已得到了理论计算的证实.
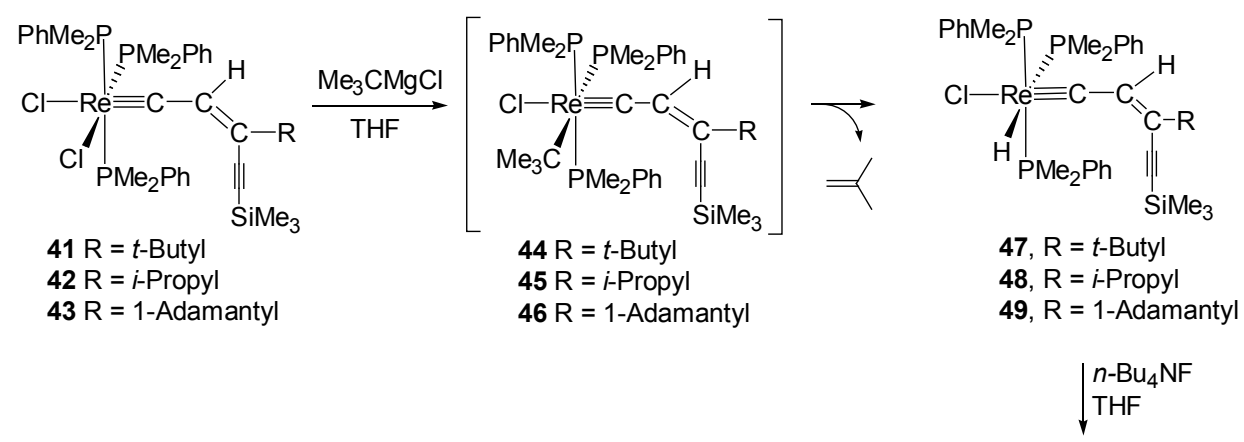

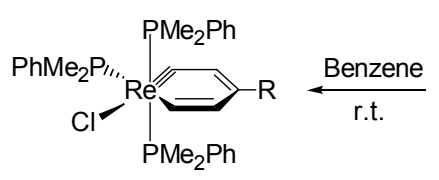

$56 \mathrm{R}=t$-Butyl $57 \mathrm{R}=i$-Propyl $58 \mathrm{R}=1$-Adamantyl

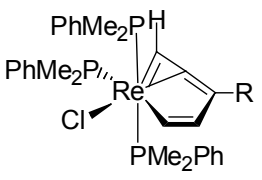

$53 \mathrm{R}=t$-Butyl

$54 \mathrm{R}=i$-Propyl

$55 \mathrm{R}=1$-Adamantyl

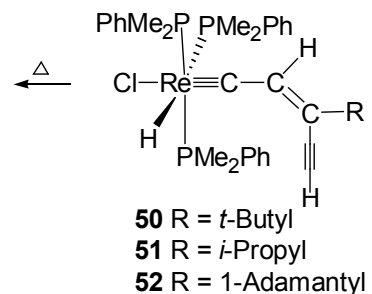

Scheme 7
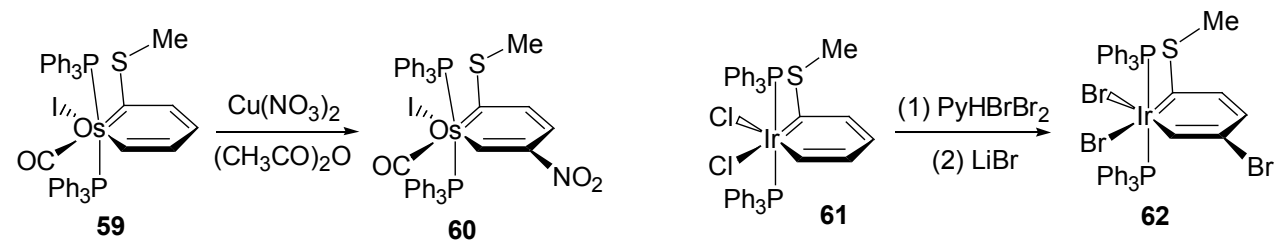

Scheme 8 


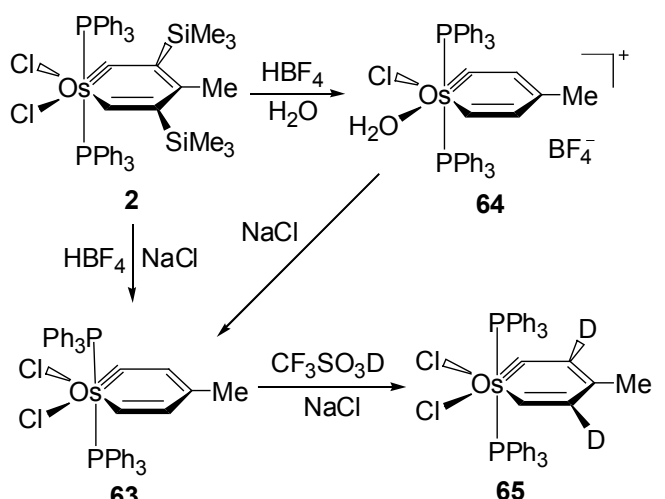

Scheme 9

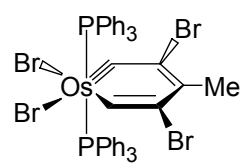

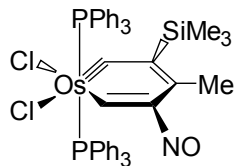

66

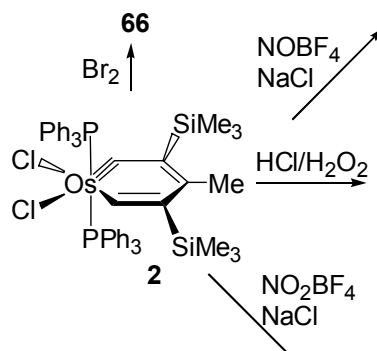
67

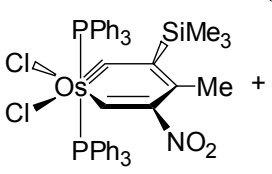

69

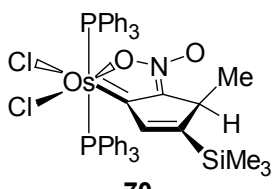

Scheme 10

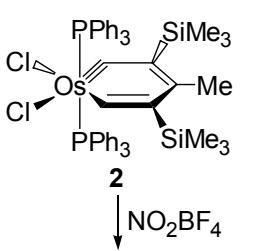

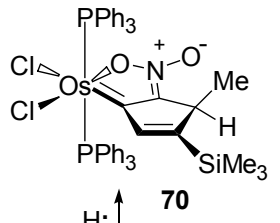<smiles>COc1cc(P(Cl)(Cl)(Cl)c2ccccc2)cc([N+](=O)[O-])c1[N+](=O)[O-]</smiles>

71

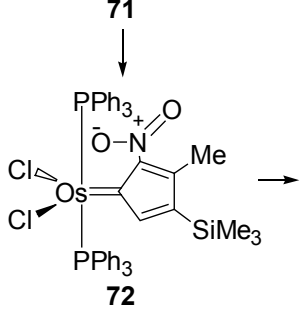

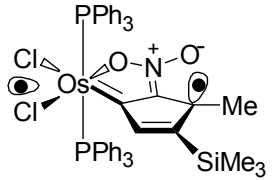

74

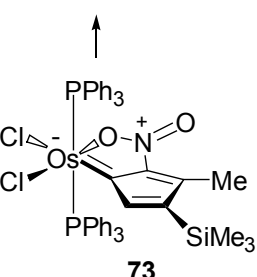

73

\section{Scheme 11}

锇苯炔化合物 2 和 $\mathrm{AlCl}_{3} / \mathrm{H}_{2} \mathrm{O}_{2}$ 反应得到了含氯化产 物 68 和 75 的混合物(Scheme 12). 化合物 68 和 75 的相
对量可以通过控制原料 $2, \mathrm{AlCl}_{3}$ 和 $\mathrm{H}_{2} \mathrm{O}_{2}$ 三者的比例来调 节. 当 $2: \mathrm{AlCl}_{3}: \mathrm{H}_{2} \mathrm{O}_{2}$ 的比例为 $1: 21: 25$ 时, 反应的 主要产物是化合物 68; 而当 $2: \mathrm{AlCl}_{3}: \mathrm{H}_{2} \mathrm{O}_{2}$ 的比例为 $1: 5: 10$ 时, 得到的主要产物则为化合物 75 .

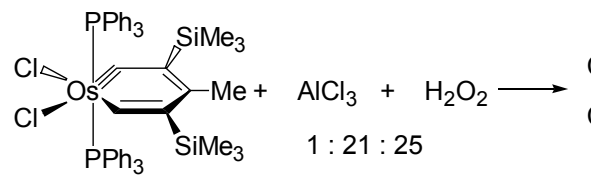

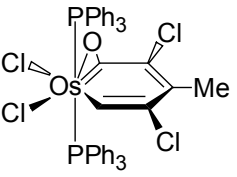
2 $68(50 \%)$

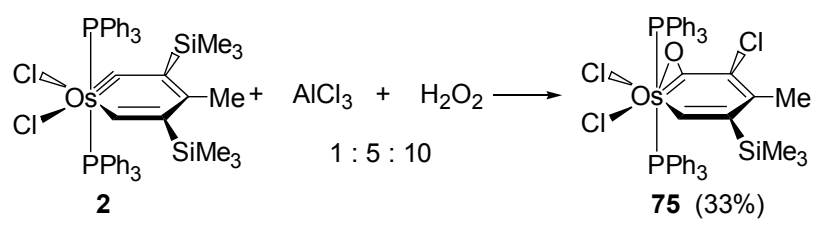

Scheme 12

锇苯炔化合物化合物 63 也能和亲电试剂, 如 $\mathrm{Br}_{2}$ 、 $\mathrm{NO}_{2} \mathrm{BF}_{4}, \mathrm{HCl} / \mathrm{H}_{2} \mathrm{O}_{2}$ 和 $\mathrm{AlCl}_{3} / \mathrm{H}_{2} \mathrm{O}_{2}$ 发生亲电取代反应. 如 Scheme 13 所示, 锇苯炔 63 和过量的 $\mathrm{Br}_{2}$ 反应得到的主 要产物是溴化产物 66. 在 $\mathrm{NaCl}$ 存在下, 溶于 $\mathrm{CH}_{2} \mathrm{Cl}_{2}$ 中 的化合物 63 和 $\mathrm{NO}_{2} \mathrm{BF}_{4}$ (1:4 物质的量的比)在 $-6 \sim 5$ ${ }^{\circ} \mathrm{C}$ 间反应 $2 \mathrm{~h}$ 得到了一个混合物, 从这个混合物中可分 离出含 $\mathrm{NO}_{2}$ 取代基的锇苯炔化合物 76. 化合物 63 与 $\mathrm{HCl} / \mathrm{H}_{2} \mathrm{O}_{2}$ 或 $\mathrm{AlCl}_{3} / \mathrm{H}_{2} \mathrm{O}_{2}$ 反应生成了氯化产物 68. 理论 计算表明亲电取代反应的区域选择性是由前线轨道所 控制的 ${ }^{[30]}$.

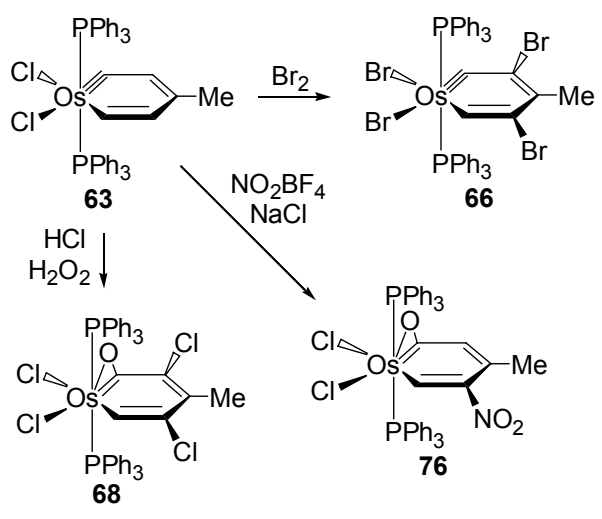

Scheme 13

\section{2 亲核加成反应}

锇苯炔化合物 77 也能和亲核试剂反应. 在 $\mathrm{K}_{2} \mathrm{CO}_{3}$ 存在下，化合物 77 同甲醇反应得到了锇苯化合物 78 (Scheme 14) ${ }^{[32]}$. 化合物 77 还能和水反应得到锇苯化合 物 79. 化合物 79 可发生水解反应形成化合物 80 .

锇苯炔化合物 77 与 $\mathrm{NaBH}_{4}$ 反应得到的是环成二烯 配合物 81 (Scheme 15), 而不是预期的金属苯化合物. 81 可能是经如下机理而形成的: 首先 $\mathrm{H}^{-}$进攻锇苯炔 77 的 


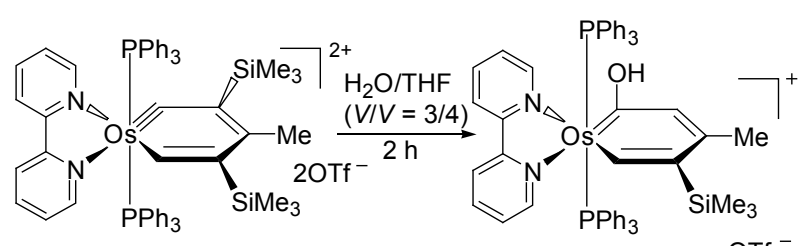

77

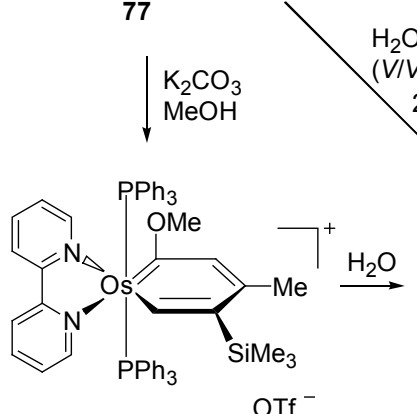

78

OTf $^{-}$
$\mathrm{H}_{2} \mathrm{O} / \mathrm{THF}$ $V / V=1 / 15)$

79 OTf $\mathrm{H}_{2} \mathrm{O}$

\section{Scheme 14}

卡拜碳得到锇苯中间体 82,82 可发生还原消除反应得到 $\eta^{1}$-环伐二烯配合物 83,83 再重排而最终得到 $\eta^{5}$-环成二 烯配合物 81. 这个机理与理论计算的结果是吻合的.

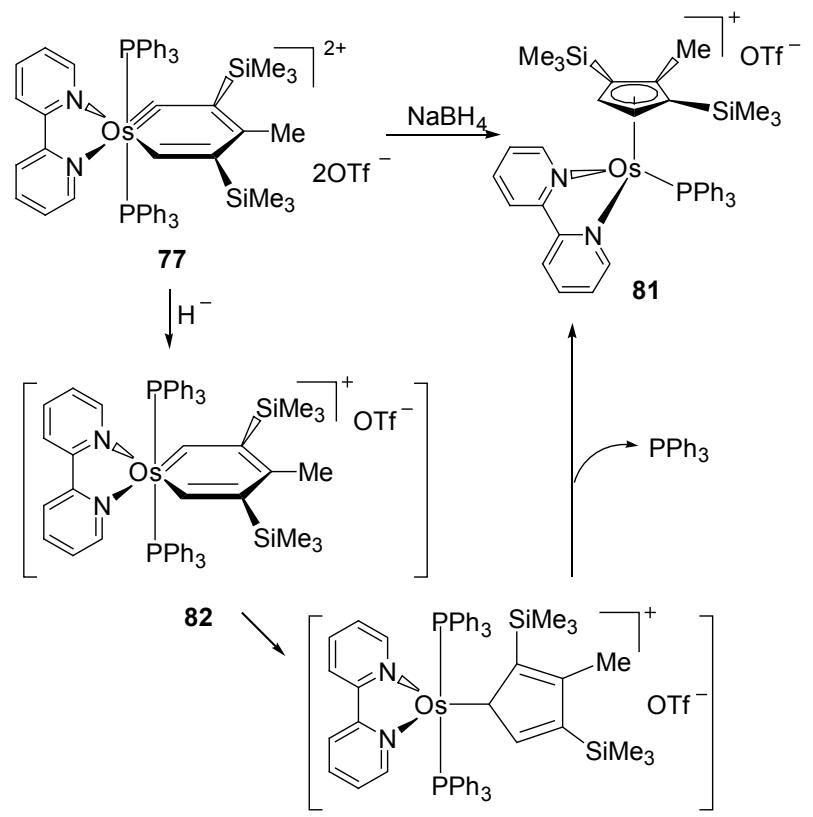

83

Scheme 15

最近, 夏海平等 ${ }^{[33}$ 报道了锇苯炔化合物 20 和亲核 试剂的反应. 锇苯炔化合物 20 分别和乙基锂、甲硫醇钠 反应得到了异锇苯化合物 84 和 85 (Scheme 16). 有趣的 是, 化合物 20 和伯胺反应得到的是开环产物(如 86 和 87) ${ }^{[22]}$.

\section{3 配体取代反应}

锇苯炔也可以发生配体取代反应而形成含不同配 体的锇苯炔. 例如, 锇苯炔 2 在 TlOTf 存在时与 $2,2^{\prime}$-联 吡啶反应生成了锇苯炔 77; 它与 2 equiv. 的 $\mathrm{HBF}_{4}$ 在湿的
二氯甲烷中反应得到了锇苯炔化合物 88 (Scheme 17) ${ }^{[32]}$.

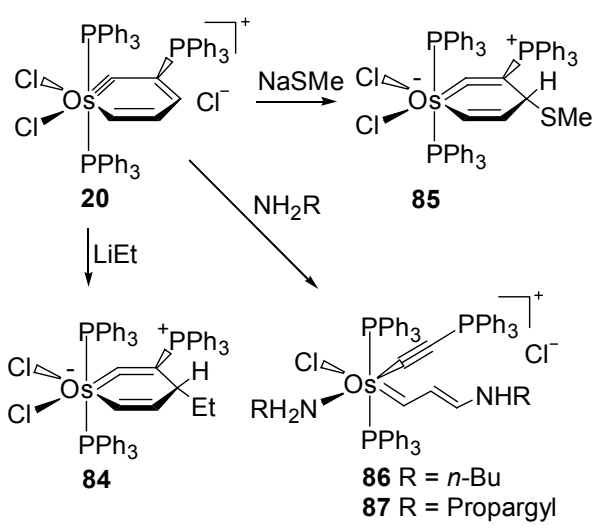

Scheme 16

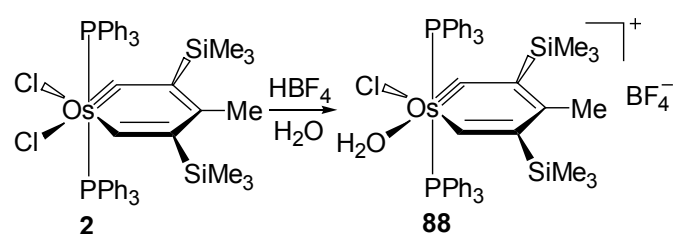

2

88

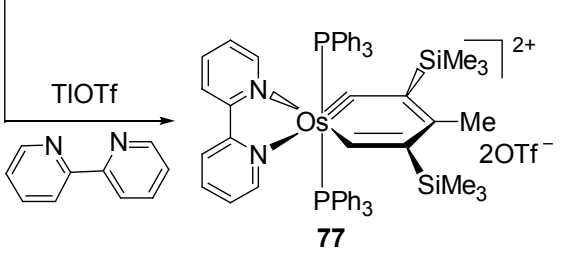

Scheme 17

\section{4 转化成过渡金属卡宾化合物}

金属苯可发生还原消除反应而形成环戊二烯配合 物. 原理上, 过渡金属苯炔也可能发生还原消除反应得 到卡宾化合物, 然而, 这类反应却很少被观察到. 2011 年, 我们研究组 ${ }^{[24]}$ 发现锇苯炔化合物 32 和 36, 在溶液 中呈现热力学不稳定性, 它们会发生还原消除反应而生 成相应的锇卡宾化合物 89 和 90 (Eq. 1).

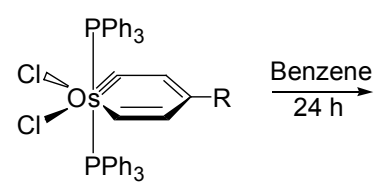

$32 \mathrm{R}=t$-Butyl $36 \mathrm{R}=1$-Adamantyl<smiles>[R]c1ccc(C(Cl)(Cl)c2ccccc2)c(P(Cl)(Cl)(Cl)Cl)c1</smiles>

$89 \mathrm{R}=t$-Butyl $90 \mathrm{R}=1$-Adamantyl
值得一提的是，在类似的条件下，相关的锇苯炔化 合物 $\mathrm{Os}\left\{\equiv \mathrm{CCH}=\mathrm{C}\left(\mathrm{CMe}_{3}\right) \mathrm{CH}=\mathrm{CH}(n\right.$-pentyl $\left.)\right\} \mathrm{Cl}_{2}$ $\left(\mathrm{PPh}_{3}\right)_{2} \quad$ (31), $\mathrm{Os}\{\equiv \mathrm{CCH}=\mathrm{C}(1$-adamantyl $) \mathrm{CH}=\mathrm{CH}(n-$ propyl $)\} \mathrm{Cl}_{2}\left(\mathrm{PPh}_{3}\right)_{2}$ (35)及 $\mathrm{Os}\{\equiv \mathrm{CCH}=\mathrm{CMeCH}=\mathrm{CH}\} \mathrm{Cl}_{2}-$ $\left(\mathrm{PPh}_{3}\right)_{2}(63)$ 是稳定的, 它们不会发生还原消除反应而生 成相应的卡宾化合物. 理论计算结果表明，锇苯炔化合 物 31, 32, 35, 36 及 63 稳定性的不同是由于取代基的立 


\section{体效应所造成的.}

\section{3 总结与展望}

金属苯炔化合物具有丰富的化学. 目前已成功合成 了含锇和锞的金属苯炔. 实验和理论研究都表明这些金 属苯炔具有芳香性. 它们既可以发生芳香体系的经典反 应(如亲电取代反应), 也可以发生金属有机化合物的反 应(如卡宾化合物的形成). 金属苯炔的化学仍有诸多新 的挑战和机遇，特别是如何来合成含其它金属的金属苯 炔，以及对它们性能的研究和应用的拓展.

\section{References}

[1] See for example: (a) Balaban, A. B. Top. Heterocycl. Chem. 2008, 19, 203.

(b) Jutzi, P. Angew. Chem., Int. Ed. Engl. 1975, 14, 232.

[2] Märkl, G. Angew. Chem., Int. Ed. Engl. 1966, 5, 846

[3] (a) Ashe III, A. J. Acc. Chem. Res. 1978, 11, 153. (b) Ashe III, A. J. Top. Curr. Chem. 1982, 105. 125.

[4] (a) Kinjo, R.; Ichinohe, M.; Sekiguchi, A.; Takagi, N.; Sumimoto, M.; Nagase, S. J. Am. Chem. Soc. 2007, 129, 7766.

(b) Tokitoh, N.; Shinohara, A.; Matsumoto, T.; Sasamori, T.; Takeda, N.; Furukawa, Y. Organometallics 2007, 26, 4048.

(c) Wakita, K.; Tokitoh, N.; Okazaki, R.; Nagase, S. Angew. Chem., Int. Ed. 2000, 39, 634 .

(d) Wakita, K.; Tokitoh, N.; Okazaki, R.; Takagi, N.; Nagase, S. J. Am. Chem. Soc. 2000, 122, 5648.

[5] For recent reviews on aromatic species containing a heavier group 14 element, see:

(a) Wang, Y. Z.; Robinson, G. H. Organometallics 2007, 26, 2.

(b) Lee, V. Y.; Sekiguchi, A. Angew. Chem., Int. Ed. 2007, 46, 6596.

(c) Tokitoh, N. Acc. Chem. Res. 2004, 37, 86.

(d) Tokitoh, N. Bull. Chem. Soc. Jpn. 2004, 77, 429.

[6] Examples of recent work:

(a) Mizuhata, Y.; Noda, N.; Tokitoh, N. Organometallics 2010, 29, 4781 .

(b) Mizuhata, Y.; Sasamori, T.; Nagahora, N.; Watanabe, Y.; Furukawa, Y.; Tokitoh, N. Dalton Trans. 2008, 4409.

(c) Mizuhata, Y.; Sasamori, T.; Takeda, N.; Tokitoh, N. J. Am. Chem. Soc. 2006, 128, 1050.

[7] Ashe III, A. J.; Shu, P. J. Am. Chem. Soc. 1971, 93, 1804.

[8] (a) Zhu, Z.; Wang, X.; Olmstead, M. M.; Power, P. P. Angew.
Chem., Int. Ed. 2009, 48, 2027.

(b) Ashe III, A. J.; Al-Ahmad, S.; Kampf, J. W. Angew. Chem., Int. Ed. Engl. 1995, 34, 1357.

[9] Thorn, D. L.; Hoffman, R. Nouv. J. Chim. 1979, 3, 39.

[10] Elliott, G. P.; Roper, W. R.; Waters, J. M. J. Chem. Soc., Chem. Commun. 1982, 811.

[11] Bleeke, J. R. Chem. Rev. 2001, 101, 1205.

[12] He, G.; Xia, H.; Jia, G. Chin. Sci. Bull. 2004, 49, 1543.

[13] Wright, L. J. J. Chem. Soc., Dalton Trans. 2006, 1821.

[14] Landorf, C. W.; Haley, M. M. Angew. Chem., Int. Ed. 2006, 45, 3914.

[15] Bleeke, J. R. Acc. Chem. Res. 1991, 24, 271.

[16] Bleeke, J. R. Acc. Chem. Res. 2007, 40, 1035.

[17] Roper, W. R. Angew. Chem., Int. Ed. 2001, 40, 2440.

[18] Wen, T. B., Zhou, Z. Y.; Jia, G. Angew. Chem., Int. Ed. 2001, 40, 1951.

[19] Jia, G. Acc. Chem. Res. 2004, 37, 479.

[20] Jia, G. Coord. Chem. Rev. 2007, 251, 2167.

[21] Wen, T. B. Hung, W. Y.; Sung, H. H. Y.; Williams, I. D.; Jia, G. J. Am. Chem. Soc. 2005, 127, 2856.

[22] Zhao, Q.; Zhu, J.; Huang, Z. A.; Cao, X. Y.; Xia, H. Chem.-Eur. J. 2012, 18, 11597 .

[23] He, G.; Zhu, J.; Hung, W. Y.; Wen, T. B.; Sung, H. H. Y.; Williams, I. D.; Lin, Z.; Jia, G. Angew. Chem., Int. Ed. 2007, 46, 9065.

[24] Chen, J.; Shi, C.; Sung, H. H. Y.; Williams, I. D.; Lin, Z.; Jia, G. Angew. Chem., Int. Ed. 2011, 50, 7295.

[25] Liu, B.; Xie, H.; Wang, H.; Wu, L.; Zhao, Q.; Chen, J.; Wen, T. B.; Cao, Z.; Xia, H. Angew. Chem., Int. Ed. 2009, 48, 5461.

[26] Chen, J.; Shi, C.; Sung, H. H. Y.; Williams, I. D.; Lin, Z.; Jia, G. Chem. Eur. J. 2012, 18, 14128.

[27] Chen, J.; Sung, H. H. Y.; Williams, I. D.; Lin, Z.; Jia, G. Angew. Chem., Int. Ed. 2011, 50, 10675.

[28] Rickard, C. E. F.; Roper, W. R.; Woodgate, S. D.; Wright, L. J. Angew. Chem., Int. Ed. 2000, 39, 750.

[29] Clark, G. R.; Johns, P. M.; Roper, W. R.; Wright, L. J. Organometallics 2008, 27, 451.

[30] Wen, T. B.; Ng, S. M.; Hung, W. Y.; Zhou, Z. Y.; Lo, M. F.; Shek, L. Y.; Williams, I. D.; Lin, Z. Jia, G. J. Am. Chem. Soc. 2003, 125, 884.

[31] Hung, W. Y.; Liu, B.; Shou, W.; Wen, T. B.; Shi, C.; Sung, H. H. Y.; Williams, I. D.; Lin, Z.; Jia, G. J. Am. Chem. Soc. 2011, 133, 18350.

[32] Hung, W. Y.; Zhu, J.; Wen, T. B.; Yu, K. P.; Sung, H. H. Y.; Williams, I. D.; Lin, Z.; Jia, G. J. Am. Chem. Soc. 2006, 128, 13742.

[33] Liu, B.; Xie, H.; Wang, H.; Wu, L.; Zhao, Q.; Chen, J.; Wen, T. B.; Cao, Z.; Xia, H. Angew. Chem., Int. Ed. 2009, 48, 5461. 